\title{
ON THE UNUSUALLY HIGH TEMPERATURE OF THE CLUSTER OF GALAXIES 1E 0657-56
}

Tahir Yaqoob ${ }^{1}$

$$
/ N-\because
$$

$\therefore$

Received —; accepted

${ }^{1}$ Laboratory for High Energy Astrophysics, NASA/Goddard Space Flight Center, Greenbelt, MD 20771, USA. 


\begin{abstract}
A recent $\mathrm{X}$-ray observation of the cluster $1 \mathrm{E} 06 \tau-56(z=0.296)$ with $A S C A$ implied an unusually high temperature of $\sim 17 \mathrm{keV}$. Such a high temperature would make it the hottest known cluster and severely constrain cosmological models since, in a Universe with critical density $(\Omega=1)$ the probability of observing such a cluster is only $\sim 4 \times 10^{-5}$. Here ve test the robustness of this observational result since it has such important in plications. We analysed the data using a variety of different data analysis methods and spectral analysis assumptions and find a temperature of $\sim 11-12 \mathrm{keV}$ in all cases, except for one class of spectral fits. These are fits in which tha absorbing column density is fixed at the Galactic value. Using simulated data for a $12 \mathrm{keV}$ cluster, we show that a high temperature of $\sim 17 \mathrm{keV}$ is artificially obtained if the true spectrum has a stronger low-energy cut-off than that for Galactic absorption only. The apparent extra absorption may be astrophysical ir origin, (either intrinsic or line-of-sight), or it may be a problem with the lov-energy CCD efficiency. Although significantly lower than previous measurements, this temperature of $k T \sim 11-12 \mathrm{keV}$ is still relatively high since only $\approx$. few clusters have been found to have temperatures higher than $10 \mathrm{keV}$ and the data therefore still present some difficulty for an $\Omega=1$ Universe. Our results will also be useful to anyone who wants to estimate the systematic errors involved in different methods of background subtraction of ASCA data for sources vith similar signal-to-noise to that of the $1 \mathrm{E} 0657-56$ data reported here.
\end{abstract}

Subject headings: galaxies: clusters: individual (1l: 0657-56)-intergalactic medium - large-scale structure of universe - X-ray $;:$ galaxies 


\section{INTRODUCTION}

The recently discovered cluster of galaxies, $1 \mathrm{E} 0657-56(z=0.296)$, was observed by ASCA (Advanced Satellite for Cosmology and Astrophysics; see Tanaka, Inoue \& Holt, 1994) and found to have an unusually high temperature of $17.4 \pm 2.5 \mathrm{keV}$ (Tucker et al. 1998; hereafter T98). A full account of this result and other properties of $1 \mathrm{E} 0657-56$ can be found in T98. Cosmologies in which the density of the Universe is critical $(\Omega=1)$ predict such a small number of clusters with this high a temperature that an $\Omega=1$ Universe is strongly disfavored by this single measurement of the temperature of 1E 0657-56 (see T98 and references therein). Since the cosmological implications are so strong, it is important to test the robustness of this observational result. So far there are only two other clusters with temperatures greater than $10 \mathrm{keV}$ (MS 1054-0321, Donahue et al. 1998; A2163. Markevitch et al. 1996; both have $k T \sim 12 \pm \sim 2 \mathrm{keV}$ ). Even for the latter objects the probability of observing $k T>10 \mathrm{keV}$ for just one source in an $\Omega=1$ Universe is less than 0.01 (as opposed to $\sim 4 \times 10^{-6}$ for $1 \mathrm{E} 0657-56$ ).

In this Letter we re-analyse the $A S C A$ data for $1 \mathrm{E} 0657-56$ using a variety of different data analysis methods and spectral analysis assumptions. The different methods of background-subtraction and spectral extraction are described in detail in $\S^{2}$ and the spectral analysis of these data is described in $\S 3$. Our conclusions are presented in $\S 4$.

\section{ASCA DATA REDUCTION}

ASCA observed 1E 0657-56 in 1996 May 10-11 for a duration of $\sim 65 \mathrm{ks}$. Since it is important that our results be reproducible, we describe the data reduction and analysis in some considerable detail. The reader is referred to Tanaka et al. (1994) for details of the instrumentation aboard ASCA. The two Solid State Imaging Spectrometers (SIS), hereafter 
SIS0 and SISl, with a bandpass of $\sim 0.5-10 \mathrm{keV}$, were operated in 2-CCD FAINT and BRIGHT modes. The two Gas Imaging Scintillators (GIS), hereafter GIS2 and GIS3, with a bandpass of $\sim 0.7-10 \mathrm{keV}$, were operated in standart PH mode. The SIS FAINT and BRIGH'T mode data were combined and the SIS energy scale was fixed using a prescription based on measurements of Cas A (Dotani et al. 1997). This corrects for the continuing decline in the CTI of the CCDs with time and is based on interpolation or extrapolation of the Cas A measurements. The last Cas A measurements incorporated into the analysis software were made about three months after the $1 \mathrm{E} 0657-56$ observation and since the CTI changes very slowly with time, the interpolation of these measurements results in a systematic uncertainty in the gain which is much less than $1 \%$ at $1 \mathrm{keV}$ (Dotani et al. 1997). Version 1.1 of the SIS response matrix generator was used in the analysis. However, no corrections were made for any possible offsets, fluctuations or distortion of the CCD dark level distribution (the latter also known as the 'RDD' effect). Such corrections (which themselves are subject to uncertainty) can only be applisd to FAIN'T mode data. This can be done with the software tool 'CORRECTRDD' which, however, as of the time of writing, still does not correct for the loss in CCD efficiency. We note that part of our objective is to reproduce the results of T98 who did not use CORRICTRDD either because a working version of it was not available at the time they did thei analysis. In 1-CCD mode (the SIS observation mode for most point sources) the RDD effect in SIS0 is negligible for data taken as late as early 1998. In addition to RDD there are two other effects which lead to a loss of low-energy efficiency which are important even in 1-CCD mode. One of these is due to on-board software mis-grading events for some setting s of the low-energy discriminator, and the origin of the other effect is not yet understood. The latter can reduce the SIS efficiency at $0.6 \mathrm{keV}$ by as much as $\sim 50 \%$. Documenta ion of these effects can be found on the World-Wide Web pages of the ASCA Guest Observer Facility (hereafter $A S C A$ GOF). This work is still very much on-going. For discussions of some calibration issues 
with respect to earlier data (but still relevant here) see Grandi et al. (1997) and Orr et al. (1998). Considering the finite energy resolution and signal-to-noise of SIS data, the loss of low energy efficiency (which seems to be worse in SIS1) can manifest itself as an apparently larger measured column density (up to $\sim$ few $\times 10^{20} \mathrm{~cm}^{-2}$ ). In 2-CCD mode, the mode relevant for this observation of 1E $0657-56$, the effect is worse in both SIS0 and SIS1. Typically, for data taken in late 1997 to early 1998 column densities measured for clusters by SISO and SIS1 can be a factor $\sim 2$ and $\sim 4$ larger than the corresponding column densities measured by the ROSAT PSPC. However, a systematic study and development of quantitative corrections is still underway (see $A S C A$ GOF Web pages).

Data were screened so that accepted events satisfied the following criteria: (i) data were taken outside the SAA; (ii) the time since or before passage through the SAA or a satellite day/night transition was $>50 \mathrm{~s}$; (iii) the elevation angle to Earth was $>5^{\circ}$; (iv) the magnetic cut-off rigidity $(\mathrm{COR})$ was $>\tau \mathrm{GeV} / \mathrm{c}$; the deviation of the satellite from the nominal pointing position was $<0.01^{\circ}$; $(v)$ the SIS parameters measuring active CCD pixels registered $<100$ active pixels per second, and (vi) the radiation-belt monitor registered $<500 \mathrm{ct} / \mathrm{s}$. Hot and flickering pixels in the SIS were removed. Screening resulted in net 'good' exposure times in the range $\sim 23.6-25.1 \mathrm{Ks}$ for the four instruments.

Source events were extracted from the GIS using circular regions with radii 6 '. For the SIS, two different types of extraction region were used: (i) circular, with radii 4' (which we call ' $\mathrm{C}$ ') and (ii) rectangular (which we call 'R'), using the exact dimensions used by T98. The reason for doing this is that no azimuthal dependence is included in the current XRT responses so it is useful to check whether there are any systematic errors introduced by using non-circular extraction regions.

Background spectra were accumulated in three different ways. The first, which we call 'B4', used released blank sky fields which were taken in 4-CCD mode. Events from the 
entire field-of-view were used, excluding the outer ring in the GIS. The second method (which we call 'LS', for local, same chip) used local background events from the same chip as the source at radii greater than 5.5' from the source centroid. For the GIS, annulii centered on the source and having inner and outer radii of $8^{\prime}$ and 12 'respectively were used. The third method (which we call 'LO' for local, off-chip) used the entire off-source SIS chip for background (slight differences in the spectral responses of the different chips are negligible compared to statistical and background-subtraction errors). For the GIS, elliptical regions with minor and major axes of 6' and 12' respectively, offset as far as possible from the source centroid, were used. All background data were subject to identical selection criteria as the on-source data. Using these different methods of background subtraction will then test the robustness of any spectral results to differences in the background in different CCD modes and SIS chips, positions on the sky, epoch, and contamination from the source itself (in the LS backgrounds). The background-subtracted spectra extracted from circular regions resulted in count rates in the range $0.21-0.32 \mathrm{ct} / \mathrm{s}$ for the four instruments, whilst the smaller, rectangular SIS regions yielded only $0.21 \mathrm{ct}$, 's for SISO and $0.14 \mathrm{ct} / \mathrm{s}$ for SIS1.

For the X-ray Telescope (XRT) effective areas we used version 2.72 of ASCAARF with both empirical effective area factors activated (these force residuals from fitting $A S C A$ data for the Crab Nebula to be less than $3 \%$ ). The 'extended source' option was used in generating the XRT effective areas. We also tried some preliminary spectral fitting with responses made using the 'point source' option and found negligible difference in the resulting source spectral parameters. From this we conc ude that systematics in the XRT responses due to the spatial extent of $1 \mathrm{E} 0657-56$ can be neglected in comparison to other sources of systematic error. 


\section{SPECTRAL FITTING RESULTS}

We fitted spectra from the four $A S C A$ instruments simultaneously in the range $0.5-10$ $\mathrm{keV}$ with a Raymond-Smith Plasma model, plus a cold, neutral absorber at $z=0$. A total of three free, interesting parameters were involved (the plasma temperature, $k T$, plasma abundances relative to solar, $A / A_{\odot}\left(\mathrm{Fe} / \mathrm{H}=4.68 \times 10^{-5}\right)$, and the column density, $N_{H}$. The four independent instrument normalizations were also free but the deviation of any of the four normalizations from their mean was less than $20 \%$. Such a large systematic difference between fluxes measured by the SIS and GIS is common for extended sources with photon-limited spectra. Hereafter we quote mean fluxes and luminosities from the two SIS, which agree with each other to better than $1 \%$, and are known to be more reliable than GIS normalizations. Note that the Galactic value of the column density, obtained from Dickey and Lockman (1990) is $6.55 \times 10^{20} \mathrm{~cm}^{-2}$.

First we tested the effect of using the three different methods of background subtraction (see §2) for circular source extraction regions. The results are shown in Table 1 (fits 1-3). The ratios of the data to the best-fitting model for fit \#3 is shown in Figure 1. For all three fits we obtain $k T \sim 11-12 \mathrm{keV}, N_{H} \sim 15 \times 10^{20} \mathrm{~cm}^{-2}$, and $A / A_{\odot} \sim 0.20$. For all three parameters, the systematic differences arising from the different methods of background subtraction are less than the statistical errors (see Table 1). From fit \#1 we obtain a 0.5-10 $\mathrm{keV}$ observed flux and intrinsic luminosity of $1.3 \times 10^{-11} \mathrm{erg} \mathrm{cm}^{-2} \mathrm{~s}^{-1}$ and $5.6 \times 10^{45} \mathrm{erg} \mathrm{s}^{-1}$ respectively $\left(H_{0}=50 \mathrm{~km} \mathrm{~s}^{-1} \mathrm{Mpc}^{-1}\right.$ and $\left.q_{0}=0\right)$. Similar values were obtained for the remaining spectral fits.

Next we tested the effect of using rectangular extraction regions for the SIS spectra (as in T98), using the exact dimensions as used by T98 (fit \#4) and obtained very similar parameters to fits 1-3, except for a slightly lower column density. Note that the background and region extractions for fit \#4 are the closest in similarity to those used by T98. Thus 
neither the different background spectra nor different ex1 raction regions can account for the significantly lower temperatures that we obtain. Note tlat our $90 \%$ confidence regions do not overlap with the T98 measurements of $17.4 \pm 2.5 \mathrm{keV}$.

We then investigated the effect of fixing $N_{H}$ at the Galactic value. The results for fit \#5 (see Table 1) pertain to the use of the 'LS' background ( $\$ 2$ ) and circular source extraction. The background ('B4') and extraction regions (' $R$ ') for fit \#6 are similar to those used by T98. It can be seen that the resulting values of $k T$ are now in agreement with T98. The ratios of data to best-fitting model for fit \#5 are shown in Figure 2 in which the deficit at low energies is clearly seen in the data (the fit $i$; only marginally acceptable at the $90 \%$ confidence level). This deficit is not seen in the data presented by T98 because they used smaller spectral extraction regions (as in fit \#6) so their statistical errors were larger. Note that T98 do not explicitly state whether the absorption was fixed at the Galactic value, nor do they give the actual value used, if it was.

We can easily test whether the high temperature of $\sim 17 \mathrm{keV}$ is an artifact of fixing the column density at the Galactic value when the actual data clearly require a stronger low-energy turnover (Figure 2). We simulated a $40 \mathrm{ks}$ exposure SISO spectrum using an input model consisting of a Raymond-Smith Plasma with $k T=12 \mathrm{keV}, N_{H}=14 \times 10^{20} \mathrm{~cm}^{-2}$, and the same flux as the real $1 \mathrm{E}, 0657-56$ data, and abu idances of 0.2 relative to solar. We then fitted the simulated data with the same model but with the column density fixed at the Galactic value and all other parameters free. We obtained a best-fitting temperature of $17.6_{-2.6}^{+3.0} \mathrm{keV}$. This clearly demonstrates that if the cclumn density in fitting the real IE $0657-56$ data is fixed when the data really have a st onger low-energy cut-off (whether or not this is due to extra intrinsic absorption or a calit.ration problem is irrelevant) the result is an artificially high temperature. Further proof of this comes from excluding the low-energy part of the real data for spectral fitting. We performed four-instrument fits 
in the restricted energy band $1.0-10 \mathrm{keV}$, with only Galactic absorption included. This significantly reduces the impact of any low-energy turnover. We used background spectra ('B4') and extraction regions ('R') most closely resembling those of T98 and the results are shown in Table 1, fit \#7. We obtain a lower temperature of $k T=13.8_{-1.9}^{+2.2} \mathrm{keV}$. We found that excluding even more of the low-energy data reduced the measured temperature even further.

The next spectral fit in Table 1 (fit \#8) uses SIS spectra made from only single-pixel CCD events (grade 0) in order to investigate the effect on the measured temperature of the less uncertain calibration of two-pixel events (under some circumstances - see the ASCA GOF Web pages). Table 1 shows that the best-fitting temperature is still $\sim 12 \mathrm{keV}$.

The basic ASCA event data used in T98 were produced from the raw data using 'Revision 1' (REV1) of the processing pipeline software. The event data we used was produced later, using 'Revision 2' (REV2) of that software (REV1 was phased out). The final spectral fit in Table 1 (fit \#9) was performed to check whether the difference between REV1 and REV2 data could be responsible for the unusually high cluster temperature measured by T98. In practice the only differences between REV1 and REV2 are minor updates in the calibration files for both SIS and GIS that are used to compute temporal gain corrections. For the SIS, REV2 uses data from new calibration observations not available at the time REV1 was used (as opposed to extrapolation using old data). For the GIS, REV2 incorporates temporal gain corrections not applied in REV1. Another change in REV2 data is that pre-screened events files are made using different screening criteria than those used for REV1. However, this latter difference is not relevant to us because we dot use pre-screened event data and nor did T98. Fit \#9 in Table 1 is essentially a repeat of fit \#1 except that fit \#9 uses REV1 data and fit \#1 uses REV2 data. It can be seen that the resulting spectral parameters for REV1 and REV2 are indistinguishable. 


\section{SUMMARY AND CONC:LUSIONS}

We have re-analysed the $A S C A$ data for the cluster of galaxies $1 E 0657-56$ using a variety of different data analysis and spectral fitting assumptions. The actual methods of data extraction and background subtraction cover the entire range of methods used, plus more, in the majority of the hundreds of papers published using ASCA data. The one method of extraction and analysis used by Tucker $e$ al. (1998) yielded the unusually high temperature of $k T=17.4 \pm 2.5 \mathrm{keV}$. All but one of our methods of analysis gave a much lower temperature, $\sim 11-12 \mathrm{keV}$. The only way we could reproduce the higher temperature was to fix the column density at the Galactic value, even though this leads to a low-energy deficit in the data, which clearly require a stronger low-energy cut-off. We proved that fixing the column density in this way leads o an artificially high temperature

by simulating $A S C A$ data for a cluster with $k T=12$ kel and a column density higher than Galactic, and then re-fitting the data with the column fixed at the Galactic value. The additional low-energy cut-off in the real $1 \mathrm{E} 0657-56$ da a may be astrophysical in origin (intrinsic or line-of-sight absorption) or it may be due to as yet uncalibrated loss of CCD efficiency in the SIS. The latter is a very difficult problem because there are a number of time-dependent effects which are causing a loss in the lo $v$-energy CCD efficiency and these are under on-going investigation by the $A S C A$ GOF and $A S C A$ instrument teams.

Even aside from calibration problems, it is recomm snded that the absorbing column density is not fixed at the Galactic value when spectral fitting $A S C A$ data. This is because doing so assumes there is no other photo-electric absorp ion, either along the line-of-sight, or intrinsic. If this assumption is wrong then, as we have shown, one obtains artificially high cluster temperatures. Besides, such a spectral fittin assumption allows no room for systematic errors in the actual value for Galactic absor, tion. Even if there is no other absorption and no calibration problem, an error in the Galactic column density will lead to 
an error in the measured temperature.

We note that our results will also be useful to anyone who wants to estimate the systematic effects of different background-subtraction methods for ASCA data for any source with a similar count-rate to that of $1 \mathrm{E} 0657-56$ during the observation reported here $(\sim 0.21-0.32 \mathrm{ct} / \mathrm{s})$.

The temperature of $\sim 11-12 \mathrm{keV}$ for $1 \mathrm{E} 0657-56$ still puts it amongst the handful of clusters with $k T>10 \mathrm{keV}$, along with MS 1054-0321 (Donahue et al. 1998) and A2163 (Markevitch 1996). As such, their existence still poses a problem for a Universe with $\Omega=1$ in that the probability of observing only one of these clusters is $<0.01$.

The author thanks T. Dotani, Richard Mushotzky and Una Hwang for valuable discussions, the anonymous referee for some extremely helpful comments, and the $A S C A$ mission operations team at ISAS, Japan, and all the instrument teams for their dedication and hard work in making these $A S C A$ observations possible. This research made use of the HEASARC archives at the Laboratory for High Energy Astrophysics, NASA/GSFC. 
Table 1. Raymond-Smith Plasma Spectral Fits to 1E 0657-56

\begin{tabular}{lcccccc}
\hline \hline Fit \# & BGD $^{a}$ & Region $^{b}$ & $N_{H}\left(10^{20} \mathrm{~cm}^{-2}\right)$ & $k T(\mathrm{keV})$ & $A / A_{\odot}^{c}$ & $\lambda^{2} /$ d.o.f. $^{d}$ \\
\hline 1 & & & & & & \\
2 & $\mathrm{LS}$ & $\mathrm{C}$ & $14.9_{-2.5}^{+3.5}$ & $11.7_{-1.4}^{+2.2}$ & $0.20_{-0.13}^{+0.14}$ & $726.4 / 769$ \\
3 & $\mathrm{LO}$ & $\mathrm{C}$ & $15.2_{-2.3}^{+2.6}$ & $11.4_{-1.3}^{+2.0}$ & $0.20_{-0.13}^{+0.13}$ & $745.0 / 769$ \\
4 & $\mathrm{~B} 4$ & $\mathrm{C}$ & $15.1_{-2.2}^{+2.3}$ & $11.0_{-1.3}^{+1.5}$ & $0.21_{-0.11}^{+0.13}$ & $748.4 / 769$ \\
5 & $\mathrm{~B} 4$ & $\mathrm{R}$ & $13.3_{-2.7}^{+2.7}$ & $11.4_{-1.5}^{+2.1}$ & $0.24_{-0.15}^{+0.14}$ & $637.9 / 689$ \\
6 & $\mathrm{LS}$ & $\mathrm{C}$ & $6.55 \mathrm{fixed}$ & $17.6_{-2.2}^{+2.2}$ & $0.20_{-0.18}^{+0.18}$ & $814.1 / 769$ \\
7 & $\mathrm{~B} 4$ & $\mathrm{R}$ & $6.55 \mathrm{fixed}$ & $16.3_{-2.1}^{+2.4}$ & $0.22_{-0.20}^{+0.18}$ & $670.2 / 690$ \\
8 & $\mathrm{~B} 4(1-10 \mathrm{keV})$ & $\mathrm{R}$ & $6.55 \mathrm{fixed}$ & $13.8_{-1.9}^{+2.2}$ & $0.26_{-0.18}^{+0.23}$ & $577.0 / 649$ \\
9 & $\mathrm{LS}(\mathrm{G} 0)$ & $\mathrm{C}$ & $11.8_{-2.7}^{+2.8}$ & $11.5_{-1.6}^{+2.4}$ & $0.16_{-0.16}^{+0.15}$ & $708.8 / 703$
\end{tabular}

Note. - Errors are $90 \%$ confidence, corresponding tc $\Delta \chi^{2}=6.251$ or $\Delta \chi^{2}=4.605$, for three or two free parameters respectively (apart from normalizations).

${ }^{a} \mathrm{LS}=$ Local background (partial annulus) from sam' SIS chip as source; LO = local background from off-source SIS chip; B4 = standard :-CCD mode, released, blank-sky background; $\mathrm{G} 0=$ same as fit (1) but using grade 0 da a only (see text for details); 'Rev 1 ' = same as fit (1) but using 'Revision 1' processed A 4 CA data as opposed to 'Revision $2 \%$ (see text for details).

${ }^{b} \mathrm{C}=$ circular source extraction region; $\mathrm{R}=$ rectangular source extraction region with dimensions used by T98.

c Abundances relative to solar $\left(\mathrm{Fe} / \mathrm{H}=4.68 \times 10^{-5}\right)$.

${ }^{d}$ d.o.f. $=$ degrees of freedom. 


\section{REFERENCES}

Dickey, J.M., \& Lockman, F.J. 1990, Ann. Rev. Ast. Astr. 28, 215

Donahue, M., Voit, M., Gioa, I., Luppino, G., Hughes, J. P., \& Stocke, J. T. 1998, Ap.J, 502,550

Dotani, T., et al. 1997, ASCANews, 5, 14

Grandi, P., et al. 1997, A\&A, 325, L17

Markevitch, M., Mushotzky, R. F., Inoue, H., Yamashita, K., Furuzawa, A., \& Tawara, Y. 1996, ApJ, 456, 437

Orr, A., Molendi, S., Fiore, F., Grandi, P., Parmar, A.N., \& Owens, A. 1997, A\&A, 324, $\mathrm{L} 77$

Tanaka, Y, Inoue, H., \& Holt, S.S. 1994, PASJ, 46, L.37

Tucker, W., et al. 1998, ApJ, 496, L5 (T98) 


\section{Figure Captions}

\section{Figure 1}

Ratio of the ASCA data for IE $0657-56(z=0.296)$ to the best-fitting Raymond-Smith model (corresponding to fit \#3 in Table 1). The best-fitting temperature in this case is $k T=11.0_{-1.3}^{+1.5} \mathrm{keV}$.

Figure 2

The result of fitting a Raymond-Smith model to the $1 \mathrm{E} 0657-56$ ASCA data with absorption fixed at the Galactic value (see text). Shown are the ratios of the $A S C A$ data to the best-fitting Raymond-Smith model (corresponding to fit \#5 in Table 1). The best-fitting temperature in this case is $k T=17.6_{-2.2}^{+2.2} \mathrm{ke}$ ?. 


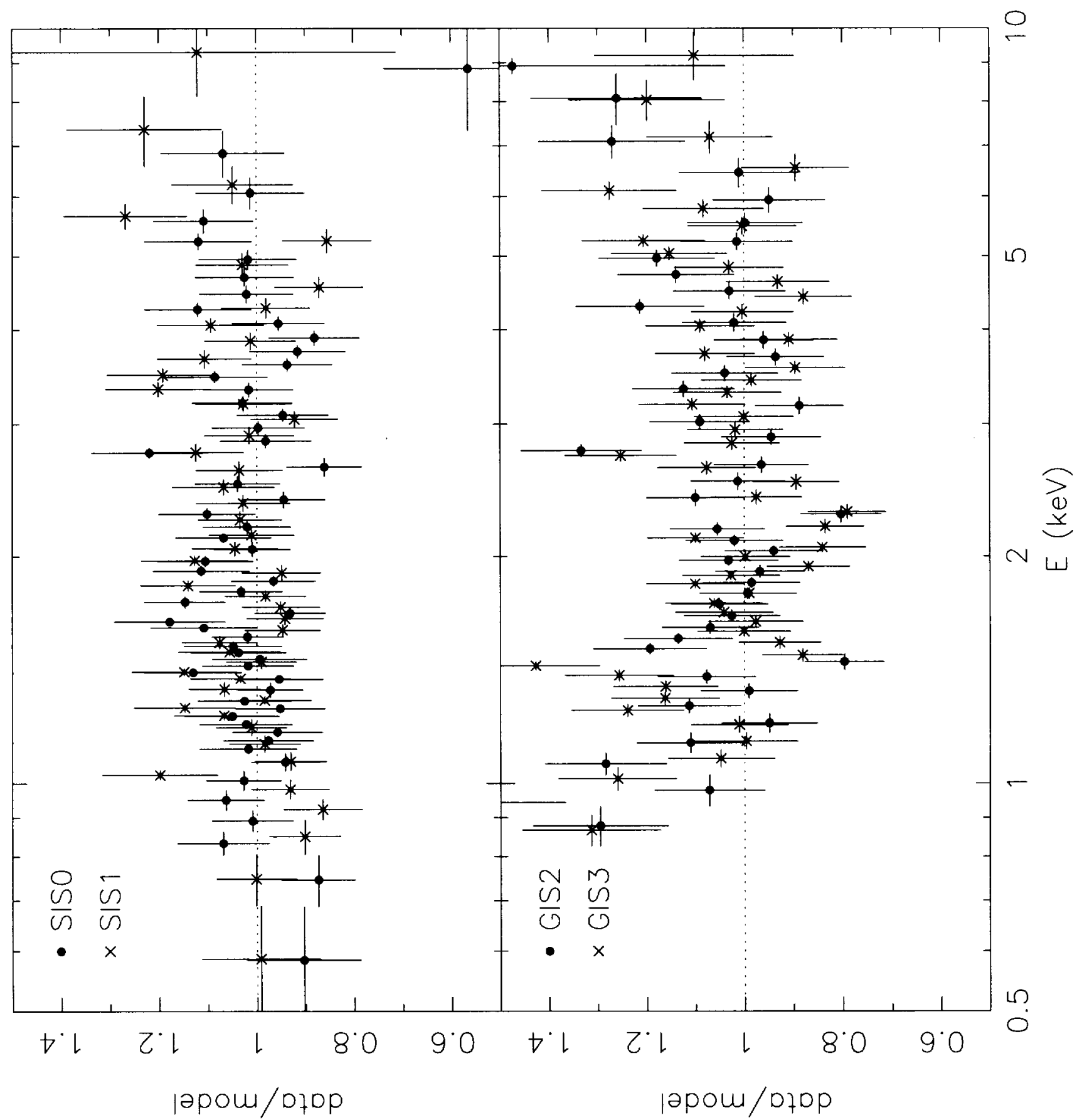





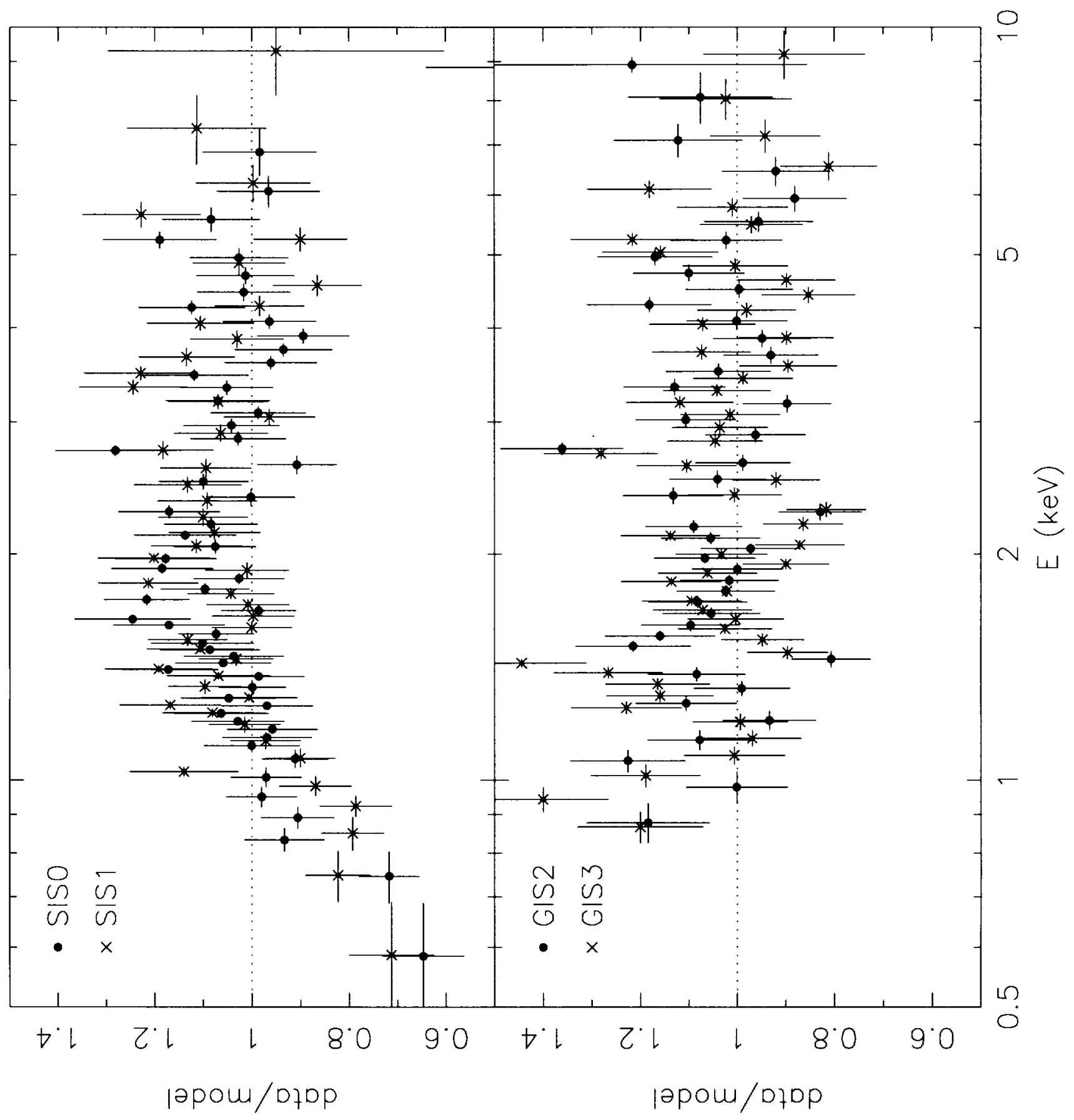


